\title{
Análise do uso de simuladores de pacientes virtuais por estudantes de Medicina
}

Analysis of the use of virtual patients simulators by medical students

\author{
1 Cristina Ribeiro Dias Barroso cristinavr95@hotmail.com \\ 1 Leonardo Silveira Gomes \\ 1 Vinícius Abrantes Silvestre \\ 1 Rafael Teixeira dos Santos \\ 1 Claudia Yamada Utagawa
}

1 Centro Universitário de Volta Redonda, UniFOA

\section{Resumo}

Os Pacientes Virtuais (PVs) são definidos como ferramentas interativas desenvolvidas em computador que simulam situações clínicas em ambiente virtual, podendo ser encontrados em vários sítios eletrônicos,possuindo diversas qualidades. Dada a importância do uso de metodologias de ensino alternativas e o impacto positivo de sua implantação na didática de ensino, o presente estudo avaliou a percepção de alunos de um curso de Medicina quanto à utilização dos PVs como ferramenta de aprendizagem e aperfeiçoamento da prática médica.

\section{Palavras-chave}

Educação médica. Simulação de paciente. Simulação por computador.

\begin{abstract}
Virtual Patients (VPs) are defined as interactive tools developed in computer that simulate clinical situations in a virtual environment. They can be found in several electronic sites and can have diverse qualities. Given the importance of the usage of alternative teaching methodologies and the positive impact of its implementation in teaching didactics, the present study evaluated medical students' perception regarding the use of VPs as a tool for learning and improving the medical practice.
\end{abstract}

\section{Keywords}

Medical education. Patient simulation. Computer simulation.

\section{Como você deve citar?}

BARROSO, Cristina Ribeiro Dias et al. Análise do uso de simuladores de pacientes virtuais por estudantes de Medicina. Cadernos UniFOA, Volta Redonda, n. 36, p. 91-100, abr. 2018. 
Análise do uso de simuladores de pacientes virtuais

por estudantes de Medicina

\section{INTRODUÇÃO}

A imposição de uma realidade cada vez mais tecnológica torna inevitável repensar o ensino, buscando a inclusão de novos recursos na formação dos alunos. Além disso, na área de saúde e, em particular, na medicina, a velocidade da produção do conhecimento científico é cada vez mais rápida, dificultando a atualização constante dos futuros médicos. A necessidade de cenários propícios à aprendizagem e à organização do cuidado em saúde tem feito com que as mudanças de conceitos dos ambientes estudantis estejam cada vez mais em evidência. À vista disso, novas metodologias de ensino surgem de forma cada vez mais rápida e, com elas, novas tecnologias educacionais (HIGGS et al., 2008).

Além disso, a dificuldade de aproximar as atividades teóricas e asexperiências clínicas que os estudantes de medicina vivenciam tem sido uma preocupação entre os educadores (HIGGS et al., 2008; CENDAN; LOK, 2012). O sistema de ensino universitário dispõe de uma estrutura que envolve longos períodos de estudos teóricos alternados com práticas clínicas, o que faz com que seja um desafio para o estudante a aplicação de todos os seus conhecimentos à prática médica (FORTE et al., 2010). 0 desenvolvimento e a utilização de tecnologias multimídia poderiam preencher a lacuna de experiência com pacientes reais, utilizando uma realidade simulada (CENDAN; LOK, 2012).

Assim, observa-se um crescimento das tecnologias da comunicação e informação que impulsionam as transformações nas mais diversas áreas do conhecimento e causam significativo impacto no processo de aprendizagem (MARIN, 2006).

Nesse âmbito, destacam-se os objetos virtuais de aprendizagem (OVAs), que são caracterizados por ambientes flexíveis de aprendizagem, sendo compatíveis com metodologias ativas de ensino-aprendizagem, valorizando a autonomia dos estudantes (COGO; SILVEIRA; CATALAN, 2006). Dessa forma, os OVAs constituem o centro de um novo paradigma de design instrucional para aprendizagem na área médica, baseados em plataformas na web (WILEY, 2000).

Nesses ambientes, destaca-se a utilização de simuladores, que são responsáveis pela promoção de competências médicas, do pensamento crítico e da resolução de problemas, fazendo com que o estudante reflita sobre suas experiências clínicas de um modo estruturado e facilitado (WILEY, 2000).

A observação de alguns contextos médicos pelos alunos, muitas vezes, não são reproduzíveis em um ambiente acadêmico, em função dos riscos ao paciente real ou do alto custo envolvido no processo (DE MORAIS; DOS SANTOS MACHADO; VALENÇA, 2013). Por esse motivo, surgem os simuladores de Pacientes Virtuais (PVs), com o objetivo de proverem um contato mais direto dos acadêmicos com situações, algumas vezes, dificilmente vivenciadas durante seu curso de graduação (SEBASTIANI, 2012). A principal característica do PV é ensinar conteúdos específicos de disciplinas ou treinar habilidades tanto teóricas como comportamentais (DE MORAIS; DOS SANTOS MACHADO; VALENÇA, 2013).

Os PVs são definidos como ferramentas interativas desenvolvidas em computador que simulam situações clínicas em ambiente virtual, podendo ser encontrados em vários sítios eletrônicos principalmente de instituições de educação médica -possuindo diversas qualidades (ASSOCIATION OF AMERICAN MEDICAL COLLEGES, 2007).

As primeiras referências encontradas de PV datam de 1991 e, desde então, seu papel na educação médica vem sendo amplamente discutido na literatura. Contudo, ao longo dos anos, o termo "Pacientes Virtuais" vem sendo inserido em diversos outros contextos, como em prontuários eletrônicos e pesquisas clínicas. Assim, a definição de PV foi consolidada ao longo dos anos como um programa de simulação do aprendizado médico com utilização de casos (ELLAWAY et al., 2006). 
A atividade apresentada pelos PVs se distancia das outras formas de aprendizado baseadas em experiências como os casos clínicos via internet que não são modificados, dependendo da resposta do aluno, as simulações com pacientes padronizados (standardised patients - SPs), os manequins e os sistemas que requerem equipamento e profissionais especializados (COOK; TRIOLA, 2009; COOK; ERWIN; TRIOLA, 2010).

Dentre as plataformas na web que possuem esse tipo de simulação, podemos citar:

- Interative Medical Cases - The New England Journal of Medicine. Disponível em: <http:// www.nejm.org/multimedia/interactive-medical-case>

- Electronic Virtual Patients - eVip. Disponível em: < http://virtualpatients.eu/>

- Virtual Patient Simulator - vpSim. Disponível em: <http://vpsim.pitt.edu/shell/CaseList_ Assignments.aspx>

- Annals Virtual Patients - American College of Physicians. Disponível em: $<$ http://vp.acponline.org/virtualpatients>

Os usuários desse tipo de programa simulam o papel de médicos ao atuarem para obter a história clínica do paciente, realizar exames físicos e solicitar e interpretar exames complementares, a fim de fazerem o diagnóstico e tomarem uma decisão terapêutica para cada caso individual (ASSOCIATION OF AMERICAN MEDICAL COLLEGES, 2007).

Os designs dos PVs são variáveis e, desse modo, podem ser classificados de acordo com diversos aspectos, como: estrutura (linear ou não linear); interatividade (como o usuário interage com os dados); forma de apresentação das informações (texto, imagem ou vídeo); progressão do caso (se a evolução depende das escolhas feitas); conhecimento do diagnóstico (se o diagnóstico é fornecido); organização dos casos (se os casos são organizados por especialidade ou dificuldade); feedback (se, ao final, é indicado algum material de apoio ou estudo); colaboratividade (individual ou em grupo); integração curricular (se faz parte de algum programa de crédito estudantil ou currículo); avaliação (se há alguma avaliação dos resultados do usuário); desenvolvimento dos casos (criação e manutenção dos PVs) e acesso (livre, mediante cadastro ou pago) (KNONOWICZ et al., 2015).

Nesse contexto, a diferença mais marcante entre os PVs é se eles são lineares ou não lineares. Isso se dá pela forma como são montadas suas estruturas. Os PV não lineares não apresentam uma sequência fixa de desenvolvimento do caso, podendo assumir caminhos e desfechos distintos, de acordo com o modo como a pessoa conduz cada situação. Ao contrário disso, os PVs lineares apresentam uma sequência fixa dos fatos, já que, caso a pessoa erre uma etapa, ela será corrigida e levada até a próxima etapa, do mesmo modo como a pessoa que a realizou corretamente.

Mesmo havendo tantas diferenças entre os PVs, Whelan afirmou, em 2014, que eles auxiliam no desenvolvimento do raciocínio clínico do estudante, principalmente se associado a um sistema de tempo para realização da simulação. Ressaltou, entretanto, que o cenário virtual ajuda pouco no desenvolvimento da empatia e habilidades comunicativas, exigindo que a instituição de ensino continue a integrar outras atividades de simulação para melhorar a relação médico-paciente.

Assim, dada a importância do uso de metodologias de ensino alternativas e o impacto positivo de sua implantação na didática de ensino, o objetivo deste estudo foi avaliar a percepção de alunos de um curso de Medicina quanto à utilização dos PV como ferramenta de aprendizagem e aperfeiçoamento da prática médica. 
Análise do uso de simuladores de pacientes virtuais

por estudantes de Medicina

\section{METODOLOGIA}

A pesquisa teve como fonte de obtenção de dados um questionário aplicado aos alunos, o qual foi vinculado a um caso clinico já existente na plataforma "Eletronic Virtual Patients (eVIP)" - disponível em: <https://labyrinth.sgul.ac.uk/openlabyrinth/mnode.asp?id=qgxlrdb1rx7jzf4jesnqdknamqajxhq > uma colaboração entre nove universidades do norte da Europa e o consórcio Medbiquitous, que serviu para avaliar a conduta clínica dos alunos, a opinião deles quanto ao uso da plataforma e uma perspectiva do impacto da possível implantação do uso de pacientes virtuais no curso de Medicina.

\subsection{Descrição do método}

O público-alvo foi os acadêmicos do $1^{\circ}$ ao $8^{\circ}$ módulos do curso de Medicina de uma faculdade da região Sul Fluminense, no interior do Estado do Rio de Janeiro, convidados a participar da pesquisa. O projeto foi aprovado pela Comissão de Ética (CAAE: 52777715.9 .0000 .5237 ) e todos os alunos voluntários assinaram o Termo de Consentimento Livre e Esclarecido (TCLE).

Um único caso clínico, pré-escolhido, intitulado "Tuberculosis, Respiratory distress syndrome, Cough", da plataforma de pacientes virtuais eVIP, foi utilizado na pesquisa. 0 caso contemplava diversos conteúdos médicos das áreas básica e clínica. Foi solicitado aos alunos que resolvessem o caso clínico durante um período determinado no laboratório de informática da instituição de ensino. Após a resolução do caso, os alunos responderam a um questionário na plataforma Google Docs. Salienta-se que os alunos acessaram a plataforma e o questionário apenas uma vez, com o bloqueio de quaisquer outros sítios eletrônicos que pudessem interferir na resolução do caso.

Para avaliar a percepção dos alunos sobre o PV, foram analisados, através de 10 afirmativas:

- O grau de interatividade de um PV previamente selecionado;

- Se a simulação auxilia no desenvolvimento de um raciocínio clínico correto pelo discente;

- Se o PV é útil para que o aluno aplique os conhecimentos aprendidos em sala de aula.

- Se a simulação contribui para demonstrar uma boa relação entre o médico e o paciente ou seus responsáveis.

Os participantes responderam a um questionário não identificado, estruturado em escala tipo Likert, de 5 pontos para cada afirmativa. A escala de Likert foi utilizada, visando mensurar o grau de concordância ou discordância dos participantes que responderam ao questionário.

Para análise dos dados obtidos, foi calculado o Ranking Médio (RM), utilizando-se o método proposto por Malhotra (2001). Através do RM, é possível verificar a concordância ou discordância das questões avaliadas, relacionando-as à frequência das respostas dos participantes.

O RM é calculado através da multiplicação, primeiro, da nota de cada item Likert (discordo totalmente $=1$, discordo parcialmente $=2$, não concordo nem discordo $=3$, concordo parcialmente $=4 \mathrm{e}$ concordo totalmente $=5$ ) pelo número de respondentes para cada item. O RM é a soma do resultado de cada item dividida pelo total de respondentes. Os valores menores que 3 obtidos por RM foram considerados discordantes e os maiores que 3 , concordantes. 0 valor exato 3 foi considerado o ponto neutro, sendo equivalente a "sem opinião" ou "indiferente". 
O questionário também coletou dados pessoais dos estudantes: sexo, idade, período da graduação eo tempo gasto para a resolução do caso (0 - 5 minutos, 6 - 10 minutos, 11 - 15 minutos, 16 - 20 minutos ou mais que 20 minutos). Após isso, cada aluno respondeu às questões referentes ao caso clínico resolvido.

Os resultados foram contabilizados e agrupados por ano da graduação $\left(1^{\circ}\right.$ e $2^{\circ}$ módulos formaram o primeiro ano, por exemplo).

Dessa forma, é demonstrado abaixo o cálculo de significância utilizado, a partir do grau de concordância, para análise do estudo:

Tabela 1 - Análise, considerando afirmativa 1, aplicada ao $1^{\circ}$ ano.

\begin{tabular}{lllllll}
\hline QUESTÃO & FREQUÊNCIA DE SUJEITOS & & & RM \\
\hline Afirmativa 1 & 5 & 4 & 3 & 2 & 1 & 2,7 \\
$\begin{array}{l}\text { O caso em questão não se mostrou eficaz na descrição } \\
\text { de um bom exame físico. }\end{array}$ & 0 & 5 & 9 & 13 & 0 & $\mathbf{2 , 7}$ \\
\hline
\end{tabular}

Fonte: dos autores, 2018.

Média Ponderada $=(5 \times 0)+(4 \times 5)+(3 \times 9)+(2 \times 13)+(1 \times 0)=73$

LogoRM $=73 /(0+5+9+13+0)=2,7$

O mesmo procedimento foi realizado com todas as afirmativas e todos os anos da graduação avaliados na pesquisa.

\section{RESULTADOS E DISCUSSÃO}

A pesquisa contou com a participação de 91 alunos, sendo 27, do primeiro ano de graduação em medicina ( $1^{\circ}$ e $2^{\circ}$ módulos); 21 , do segundo ano ( $3^{\circ}$ e $4^{\circ}$ módulos); 27 , do terceiro ano ( $5^{\circ}$ e $6^{\circ}$ módulos); e 16 , do quarto ano $\left(7^{\circ}\right.$ e $8^{\circ}$ módulos).

No que diz respeito à faixa etária, a média de idades foi de 21,8 anos entre todos os períodos, com idades variando de 18 a 39 anos. Além disso, o tempo gasto para resolução do caso teve uma média de 8,36 minutos, variando de $0-5$ até mais do que 20 minutos.

A tabela a seguir sintetiza os dados apresentados por ano da graduação:

Tabela 2 - Dados gerais, por ano da graduação.

\begin{tabular}{|c|c|c|c|c|c|}
\hline ANO DA GRADUAÇÃO & TOTAL DE ALUNOS & $\begin{array}{c}\text { ALUNOS } \\
\text { DO SEXO } \\
\text { FEMININO }\end{array}$ & $\begin{array}{l}\text { ALUNOS } \\
\text { DO SEXO } \\
\text { MASCULINO }\end{array}$ & MÉDIA DE IDADES & $\begin{array}{c}\text { MÉDIA DE TEMPO } \\
\text { GASTO PARA A } \\
\text { REALIZAÇÃOO DA } \\
\text { SIMULAÇÃO }\end{array}$ \\
\hline $1^{\circ}$ ANO & 27 & 12 & 15 & 20,1 anos & 11,05 minutos \\
\hline $2^{\circ}$ ANO & 21 & 11 & 10 & 22,5 anos & 7,5 minutos \\
\hline $3^{\circ}$ ANO & 27 & 21 & 6 & 22,4 anos & 6,74 minutos \\
\hline $4^{\circ}$ ANO & 16 & 9 & 7 & 22,8 anos & 7,68 minutos \\
\hline
\end{tabular}


Os resultados da escala Likert, assim como seu RM, estão dispostos na tabela abaixo:

Tabela 3 - Resultados dos RMs, de acordo com cada afirmativa do questionário, por ano da graduação.

\begin{tabular}{l} 
AFIRMATIVA \\
\hline AFIRMATIVA 1 \\
0 caso em questão não se mostrou eficaz \\
na descrição de um bom exame físico. \\
AFIRMATIVA 2 \\
Com o PV, pude estabelecer os exames \\
complementares que podem ser solicitados \\
em relação à hipótese diagnóstica.
\end{tabular}

AFIRMATIVA 3 TOTAL

$1^{\circ}$ ANO $\quad 2^{\circ}$ ANO

$3^{\circ}$ ANO $\quad 4^{\circ}$ ANO

GERAL

$2,6 \quad 27$

O PV não me permitiu aplicar conhecimentos de farmacologia no caso em questão.

\section{AFIRMATIVA 4}

Com o caso, aprendi a traçar um plano terapêutico para a doença.

\section{AFIRMATIVA 5}

Não foi possível reconhecer os fatores de risco para a doença em questão.

\section{AFIRMATIVA 6}

O PV apresentou uma anamnese detalhada e precisa.

\section{AFIRMATIVA 7}

O PV não me ajudou a identificar possiveis alterações no hemograma.

\section{AFIRMATIVA 8}

A simulação foi de fácil compreensão e realização, apesar de ser em uma língua estrangeira.

\section{AFIRMATIVA 9}

A simulação se mostrou útil para que eu pudesse identificar e interpretar possíveis alterações nas mais variadas funções orgânicas.

\section{AFIRMATIVA 10}

A simulação me fez reconhecer o vínculo necessário entre o médico e seu paciente, sendo o mesmo importante para o sucesso terapêutico.

$\mathbf{N}^{\circ}$ de Participantes

$\begin{array}{lllll}4,175 & 3,9 & 4,5 & 4,0 & 4,3\end{array}$

Como já foi mencionado anteriormente, a simulação foi realizada a partir do caso clínico de um PV com tuberculose pulmonar, presente na plataforma de pacientes virtuais eVIP. Vale ressaltar que a escolha do caso se deu em virtude de tal enfermidade ser uma realidade muito comum em nosso país e que teve sua incidência muito aumentada nos últimos anos, sendo imprescindível o conhecimento médico sobre $o$ assunto.

A interatividade do PV foi avaliada a partir de um tópico do questionário que requisitava que o aluno estabelecesse o grau de interatividade da simulação realizada, podendo ser alto, médio ou baixo. Do total de alunos, $58,2 \%$ classificaram a ferramenta como sendo de alto grau; e $37,4 \%$, como médio 
grau de interatividade, revelando uma interatividade expressiva, de modo a poder contribuir de maneira sólida nas atividades de aperfeiçoamento da aprendizagem dos estudantes. Tal interatividade pode ser explicada pela presença de recursos, como dados de exames complementares laboratoriais, além do dinamismo apresentado por nosso PV, visto que, por ser não linear, possibilita ao aluno que faça suas próprias escolhas e conduza o caso de sua maneira. Ademais, outros PV podem apresentar exames de imagem e vídeos, além de recursos de áudio, como áudio de bulhas cardíacas, ruídos pulmonares, entre outros.

Quando questionados quanto ao conhecimento prévio de alguma ferramenta de simulação de casos clínicos similar à apresentada, 94,5\% desconheciam a existência desse tipo de metodologia, mostrando que há necessidade de mais experimentações, divulgação e desenvolvimento de simulações no modelo dos PV, principalmente na língua portuguesa, que são muito escassos.

Para analisar se a simulação auxilia no desenvolvimento de um raciocínio clínico correto por parte do discente, foram utilizadas as afirmativas 1 e 6 , e a partir do RM, observou-se que a maioria dos alunos concordou que o PV apresenta uma boa anamnese e um bom exame físico, auxiliando um raciocínio mais facilitado e correto. A concordância entre os anos estudados se deve ao fato de o currículo do presente curso de medicina desses alunos conter cenários de prática e semiologia desde o primeiro ano da graduação. 0 contato do aluno com a prática médica desde o início auxilia no desenvolvimento de seu raciocínio clínico.

Ao julgarem se a plataforma pode ser útil para aplicar conhecimentos aprendidos em sala de aula, foram inseridas as afirmativas 2, 3, 4, 5 e 7. A maior parte dos alunos dos quatro anos estudados concordou que o PV possui informações referentes a exames complementares, farmacologia, plano terapêutico, fatores de risco, alterações em funções orgânicas e análise de hemograma. Dessa forma, pode contribuir para aplicar o conhecimento aprendido em sala de aula.

A afirmativa 8 abordou o entendimento do caso clínico, visto que era em língua inglesa. O RM foi de 3,55, indicando que a maior parte dos estudantes não demonstrou dificuldade em compreender a simulação. Tal resultado pode ser explicado pelo fato de grande parte dos alunos terem bom conhecimento de inglês ou em virtude dos comandos dos textos serem claros e de fácil entendimento ou até mesmo porque grande parte do vocabulário médico possui palavras de escrita similar, sejam elas em língua portuguesa ou inglesa.

A décima afirmativa obteve um RM de 4,175, representando que a grande maioria dos estudantes concordou parcialmente ou plenamente que o vínculo do médico com seu paciente ou com o seu familiar é importante para o sucesso terapêutico. Vale ressaltar que a grade curricular do sistema de ensino modular inclui o acadêmico de medicina no ambiente da prática clínica em Unidades Básicas de Saúde, desde o primeiro semestre da graduação. Dessa forma, o aluno é capaz de vivenciar a prática médica desde o início de sua formação, sendo a relação do médico com seu paciente um tema muito abordado nesse contexto e que deve continuar sendo estimulada.

\section{CONSIDERAÇÕES FINAIS}

A ferramenta de simulação de pacientes virtuais tem grande potencial para ser uma forma inovadora e eficaz de ensino, que, a partir de uma metodologia ativa, pode auxiliar na formação dos alunos. A grande concordância com as afirmativas propostas e aceitação dos alunos estudados valida a utilização dessa plataforma de simulação de casos clínicos virtuais, tornando interessante seu maior conhecimento pelas universidades, visando considerar a possível inserção dessa ferramenta de ensino. 
A partir das análises realizadas, é evidente que a simulação através dos PVs pode ser utilizada em todos os períodos da graduação do curso de medicina, sendo possíveis as suas diferentes inserções com base nos conhecimentos dos acadêmicos. Com a implantação cada vez maior desse tipo de ferramenta no ensino médico, será possível aos alunos o acesso a casos clínicos diversos, sendo o acadêmico capaz de aprender e aprimorar seus conhecimentos de maneira individual e autônoma.

No entanto, como qualquer outra ferramenta tecnológica, vale ressaltar que a ela deve servir como material de ensino suplementar à vivência clínica, uma vez que a relação médico-paciente é insubstituível.

Além disso, é importante frisar que, quanto antes o aluno tem acesso aos conteúdos de semiologia e ao ambiente prático da clínica médica em sua universidade, mais noção ele possui, no que diz respeito à familiarização com anamneses e exames físicos, tornando a utilização desse tipo de simulação mais fácil e útil.

\section{AGRADECIMENTOS}

Aos alunos voluntários e ao Professor Bruno Chaboli Gambarato, pela realização das análises estatísticas. 


\section{REFERÊNCIAS}

ANNALS VIRTUAL PATIENTS. American College of Physicians. Disponível em: <http://vp.acponline.org/ virtualpatients>. Acesso em: 20 maio 2016.

ASSOCIATION OF AMERICAN MEDICAL COLLEGES. Effective Use of Educational Technology in Medical Education: Summary Report of the 2006 AAMC Colloquium on Educational Technology. Washington, DC: AAMC, p. 7, 2007.

CENDAN, J.; LOK, B.The use of virtual patients in medical school curricula. Advances in physiology education, v. 36, n. 1, p. 48-53, 2012.

COGO, A.L.P.; SILVEIRA, D.T.; CATALAN, V.M. Objetos de aprendizagem digitais como ferramenta de apoio na educação em enfermagem. In: Anais do $10^{\circ}$ Congresso Brasileiro de Informática em Saúde, p. 368-9, 2006.

COOK, D.A.; ERWIN, P.J.; TRIOLA, M.M. Computerized virtual patients in health professions education: a systematic review and meta-analysis. Academic Medicine, v. 85, n. 10, p. 1589-602, 2010.

COOK, D.A.; TRIOLA, M.M. Virtual patients: a critical literature review and proposed next steps. Medical education, v. 43, n. 4, p. 303-311, 2009.

DE MORAIS, A.M.; DOS SANTOS MACHADO, L.; VALENÇA, A.M.G. Serious games na odontologia: Aplicações, características e possibilidades. In: XII Congresso Brasileiro de Informática em Saúde, Porto de Galinhas-PE. 2013.

DUFFY, F.D.; GORDON, G.H.; WHELAN, G., et al. Assessing competence in communication and interpersonal skills: The Kalamazoo 2 report. Acad Med, v. 79, p. 495-507.

ELLAWAY, R., et al. An architectural model for MedBiquitous virtual patients. Baltimore, MD: MedBiquitous, 2006.

EVIP. Eletronic Virtual Patients. Disponível em: <http://virtualpatients.eu/>. Acesso em: 20 maio 2016.

FORTE, M. et al. Portfólio Reflexivo Eletrônico na Unidade Educacional de Prática Profissional do Curso de Medicina da UFSCar. In: X Workshop de Informática Médica, Belo Horizonte. Anais do XXIX Congresso da Sociedade Brasileira de Computação. Porto Alegre: Sociedade Brasileira de Computação, p. 1566-75, 2010.

FRIEDMAN, C.P.; FRANCE, C.; DROSSMAN, D.D. A randomized comparison of alternative formats for clinical simulations. Med Decision Making, v.11, p. 265-272, 1991.

HIGGS, J. Clinical reasoning in the health professions. Elsevier Health Sciences, 2008.

HUANG G.; REYNOLDS R.; CANDLER C., Virtual patient simulation at US and Canadian medical schools. Acad Med, v.82, p. 446- 451, 2007.

INTERATIVE MEDICAL CASES. The New England Journal of Medicine. Disponível em: < http://www.nejm. org/multimedia/interactive-medical-case>. Acesso em: 20 maio 2016. 
Análise do uso de simuladores de pacientes virtuais por estudantes de Medicina

KONONOWICZ, A.A., et al. Virtual patients-what are we talking about? A framework to classify the meanings of the term in healthcare education. BMC medical education, v. 15, n. 1, p. 11, 2015.

MALHOTRA, N. Pesquisa de Marketing: uma orientação aplicada. Porto Alegre: Bookman, p. 720, 2001.

MARIN, H.F Perspectivas atuais da informática em enfermagem. Ver Bras Enferm, v. 59, n. 3, p. 354-7, 2006.

SEBASTIANI, R.L. et al. Ferramenta de autoria para construção de casos clínicos interativos para educação médica. 2012.

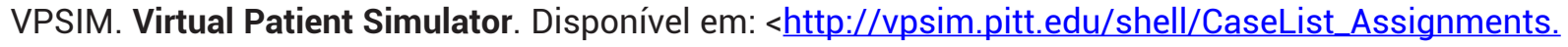
aspx>. Acesso em: 20 maio 2016.

WHELAN, A. Reforming case-based learning with non-linear gameplay: the potential of branched narratives and virtual patient models, 2014.

WILLEY, D.A. Conecting learning objects to instructional theory: A definition, a methaphor and a taxonomy. The Instructional Use of Learning Objets. 2001. 